\title{
The Effect of Training Set Timeframe on Future Performance of Machine Learning-based Malware Detection Models
}

\author{
Colin Galen \\ Capitol Technology University \\ 11301 Springfield Rd. \\ Laurel, MD 20708 \\ galencolin@gmail.com
}

\author{
Robert Steele \\ Capitol Technology University \\ 11301 Springfield Rd. \\ Laurel, MD 20708 \\ rjsteele@captechu.edu
}

\begin{abstract}
The occurrence of previously unseen malicious code or malware is an implicit and ongoing issue for all software-based systems. It has been recognized that machine learning, applied to features statically extracted from binary executable files, offers a number of promising benefits, such as its ability to detect malware that has not been previously encountered. Nevertheless it is understood that these models will not continue to perform equally well over time as new and potentially less recognizable malwares occur. In this study, we have applied a range of machine learning models to the features extracted from a large collection of software executables in Portable Executable format ordered by the date the binary was first encountered, consisting of both malware and benign examples, whilst considering different training set configurations and timeframes. We analyze and quantify the relative performance deterioration of these machine learning models on future test sets of these features, and discuss some insights into the characteristics and rate of machine learning-based malware detection performance deterioration and training set selection.
\end{abstract}

\section{Introduction}

In traditional virus detection systems, a list of known threats are maintained and regularly updated as new viruses are first encountered and identified. This has the disadvantage that new malware, will for a period not have been explicitly included in such threat lists, leading to a period of vulnerability to such new malwares after they arise.

An advantage of artificial intelligence (AI) or machine learning (ML)-based approaches to malware detection, is that they can potentially recognize malware, even when it has not been previously encountered or is not included in a list of known threats, based on models trained to probabilistically identify malware from a potentially complex combination of features present in the executable.

Nevertheless, machine learning-based approaches also face challenges in detection of new malwares. It is understood in data science practice in general that predictive models can and will decline in performance over time leading to the need for model maintenance. In the case of malware detection this decline could arise as more and increasingly novel malwares are encountered. In this paper we consider the effects of training set timeframes on model performance, in the specific context of malware detection for softwares in the very common Portable Executable (PE) binary format used on Windows and other machines.

In this paper we consider a dataset consisting of over 50,000 malware and benign software executable examples collected over a period from 2012 to 2018 [1]. For each of these examples, which corresponds to a PE format executable, over 20 static features have been extracted. An additional attribute also included is a time stamp of the date the software was first seen. In [2], the authors have analyzed the performance of machine learning models that were trained on these features, for malware detection for softwares encountered in the month following the dataset used to train the models [2]. That is, these models represent regularly re-trained models, trained on the most recently observed malwares and benign softwares.

In this work, by contrast, we consider more systematically the effect of training set period choice, and choice of model on the future performance and performance deterioration of the ML malware detection models. We quantify the relative 
performance and specifically performance deterioration of these models over time in terms of a number of evaluation metrics, including accuracy, area under the receiver operator characteristic curve (AUC), recall and precision. We consider both the performance deterioration from the end date of the training set for various models, as well as the effect of different historical durations of training sets.

The results highlight the relative rate of performance deterioration of various different types of models, suggesting the relative benefits of some forms of predictive models for robust PE-format malware detection versus others, and are also suggestive of various interesting and unanswered questions about the predictability, strengths and limitations of ML and AI for security applications such as malware detection

The remainder of the paper is structured as follows. Section 2 reviews the relevant literature, Section 3 describes the methodology used, including the dataset utilized, our data preprocessing steps, experimental setup, the experiments carried out, and the metrics used to evaluate results. Sections 4 includes the results of our experiments and Section 5 discusses the implications and research questions suggested by the results. Finally, Section 6 concludes the paper.

\section{Literature review}

A large number of previous studies have considered the application of machine learning-based predictive models to detect malware [3],[4],[5] and [6]. Many such studies have a partial limitation that they are trained and evaluated on a large set of examples, taking into account all examples regardless of date of occurrence within the sample set. In real applications, a challenge is that malwares and goodwares occurring during a later period may be different from those seen previously during the training period prior to model deployment. That is, for example a model deployed in January 2021 to detect malware, would not and could not have been trained on examples of malware including all of those first occurring up to the end of 2022 for example. As such, many evaluations of machine learning malware detection do not evaluate for any performance deterioration in the future.

At the same time, it is widely recognized in the literature and practice that machine learning models do deteriorate with time due to such phenomena as concept drift [7],[8] and in the case of malware detection this corresponds to the emergence of additional and novel malwares over time.
However, academic studies to investigate such model performance deterioration in detail over time, and the characteristics of the effect of training set selection on performance, are very rare. This may be partly due to such factors as the experiencing of deployed model performance deterioration is typically encountered and addressed in industry or applied settings where the results are not published; the real-world deterioration of models has to-date not been considered a core area of machine learning research; and also the datasets to study this phenomenon may not be readily available publicly or to researchers.

Our review of the literature indicates the significant scarceness of academic articles currently addressing this important question of quantifying malware detection model deterioration with time on real-world datasets. An initial study of such performance deterioration in relation to malware detection is only seen in the 2020 paper by the authors [9]. This scarcity is also seen in relation to measuring model deterioration over time in other application domains. Our literature review identified just one article, this being a very recent article from June 2020 [10], addressing a similar issue of model performance deterioration over time, in the case of that study in relation to models built on Medicare datasets. In the review of the literature by the authors of this 2020 article [10] they concluded "we could not find other research works that investigate the relationship between time and predictive model maintenance for big data", other than one other conference paper [11] that they identify. The study in [11] also deals with a study of maintaining predictive model performance for Medicare fraud detection. Other studies assessing the future performance of models trained on hospital length of stay [12] and mortality [13] datasets for one calendar quarter, on data from a subsequent calendar quarter, while not focusing on the problem of model maintenance per se, found little to no model performance deterioration in that domain area.

Our literature review underscores the novelty of the investigation in this current paper, but also highlights that whilst there is a large academic body of knowledge in relation to the design of novel ML and artificial intelligence algorithms, the practically significant topic of evaluating model deterioration with time has been under-addressed to-date in the academic literature. 


\section{Methods}

We utilized a data mining methodology in this work to explore the effect of training set and model selection on the future performance of malware detection predictive models. We drew our data from a published dataset [1] of the extracted features of over 50,000 malware and goodware executables. The dataset is orderable by the date each binary was first encountered.

\subsection{Data Source}

In total the dataset provides 50,123 instances of the static features extracted from software executables that consist of malware and benign software examples.

This dataset was chosen for this particular study as it has the unusual characteristic that it allows ordering of malware and goodware instances by date these have been encountered, which as noted in [2] is not an included property in many datasets previously utilized in studies of machine learning-based malware detection performance.

As described in [2], to construct the dataset, 27 features have been extracted from PE headers of these executables that were originally collected by crawling and collecting software examples over a period of 2012 to 2018. Also as per [2], of these features, 22 are numerical attributes including, base of code, base of data, characteristics, dynamic link library characteristics, file alignment, image base, machine, magic, number of relative virtual addresses and their sizes, number of sections, number of symbols, PE type, pointer-to-symbol table, size, size of code, size of headers, size of image, size of initialized data, size of optional header, size of uninitialized data, time date stamp, and entropy. A further three textual attributes include a list of dynamic libraries, functions and compilers/tools used. Of these over 50,000 instances, 21,082 instances are benign files, and the remaining 29,041 are malware, making the dataset a fairly balanced one.

\subsection{Data Preparation}

For the set of experiments performed for this paper a range of data preparation and systematic dataset segmentation activities were undertaken.

Initially, a small number of instances with invalid dates or text, such as newline characters, were manually removed. The attributes titled Magic, PE_TYPE, and SizeOfOptionalHeader were then removed because their values were identical amongst all instances in the initial dataset. Other attributes such as SHA1 and MD5 were removed because they represent unique attributes per executable and so will not benefit the training of machine learning models. Finally, the attributes TimeDateStamp and FirstSeenDate, representing dates, were treated separately as providing a mechanism to order the overall dataset into various train and validation subsets.

Once the data were preprocessed, they were ordered by the attribute FirstSeenDate, and this provides a basis to divide the dataset into groups of varying size of train and test subsets, with multiples of a group size being used to constitute model training datasets or test sets to measure the future performance of models trained on earlier occurring malware/goodware subsets.

\subsection{Experiment Setup}

Initially an open source Java-based machine learning toolkit [14] was used to preliminarily explore and visualize the performance of various base ML models on the overall dataset and randomly sampled subsets of that entire dataset. Models considered included Bayesian models such as Naive Bayes and Bayesian Networks; instance-based learners such as k-Nearest Neighbors and k-Star; rule-based learners such as JRip and PART and decision trees such as C4.5 (named J48 in this implementation), RandomTree, RandomForest and REPTree. These were trained and evaluated using 10 -fold cross validation on the entire dataset. A subset of the best performing of these were identified and selected for further consideration in the subsequent experiments. Additionally, meta-model variations of these best performing models were also trained and evaluated via 10-fold cross validation, including Bagged, Boosted, Vote, Stack and Random Committee meta versions.

Based on the inclusion of the term vectorized three textual attributes not improving or slightly decreasing model performance, we also chose to remove those three attributes from the dataset to be used.

To conduct the subsequent experiments, Python scripts were developed able to run multiple models and consider multiple training set and validation set combinations and segmentations, including in a systematic fashion. . These scripts were used to call the Java-based ML models [14] via a Python-to-Java Python wrapper package. 


\subsection{Experiments}

The first group of experiments focused on determining the rate of performance deterioration of malware detection models once trained, and then tested on future test sets. We chose the first 15,000 instances as the model training and development data subset. Models were trained and evaluated on this subset using 10 -fold cross validation to best achieve generalizable models.

The performance of these developed models was then tested on subsequently occurring subsets of 5000 instances of the overall dataset as ordered by FirstSeenDate. That is, there were seven subsequent test subsets, instances 15,001-20,000, 20,001-25,000 ..... 45,001-50,000.

The rate of future deterioration of model performance is not considered in reporting the results of almost all previous malware detection models [3-6], and so a goal of these experiments is to explore the relative rate of decline in performance of various ML models.

A second group of experiments was carried out to consider the performance of models trained on the most recently occurring software instances as ordered by time and immediately preceding the subset of software being used to test the performance of the model on. However, additionally the second group of experiments considers the effect of the length of the history of included recent instances on the future performance of the trained model. That is, for example whether all past software examples are used in model training, a mid-length history ( $3 \times$ group of 5000 ) or a short history ( $2 \times$ group of 5000). For each duration of historical training subsets used, this training is done with 10 -fold cross validation, with the performance of the trained models then further tested on the immediately following group of 5000 after the training and development subset.

\subsection{Metrics of Evaluation}

We have utilized several metrics to evaluate performance, including accuracy, area under the receiver operating characteristic curve (AUC), recall and precision.

Accuracy is the percentage of software instances correctly classified by a model. AUC is considered a good measure of discriminative performance of a model across all possible classification thresholds [15] as it represents the area under the curve of the True
Positive Rate TPR (y axis) (same as Recall) versus False Positive FPR ( $x$ axis). Typically as the classification threshold is lowered, False Positives and True Positives go up, and the AUC provides a summary measure of the model across all possible choices of classification threshold.

Recall provides a measure of what proportion of all the malwares in the test set are correctly identified by the predictive model.

Precision quantifies what proportion of all of the softwares that were predicted by the model to be malware, were actually malware.

\section{Results}

\subsection{Model Performance Deterioration Over Time}

Table 1, shows listed first, the performance of the 6 best performing models, in terms of both AUC and accuracy, when evaluated using 10-fold cross validation on the initial training set of 15,000 , and then the performance of a number of other models also trialled. The meta-model variations of the models, such as via bagging, boosting, stacking, voting and random committee were not found to improve performance over that of the base models.

TABle I. Model Performance on Training Set Evaluated Using 10-Fold Cross Validation

\begin{tabular}{|l|l|l|l|l|}
\hline ModEL & ACCURACY & AUC & PREC & RECALL \\
\hline C4.8 (J48) & 98.82 & 0.994 & 0.984 & 0.993 \\
\hline KNN (IBK) & 99.25 & 0.993 & 0.989 & 0.996 \\
\hline JRIP & 98.68 & 0.992 & 0.982 & 0.992 \\
\hline PART & 98.91 & 0.994 & 0.985 & 0.993 \\
\hline RANDOMFoREST & 99.55 & 0.999 & 0.994 & 0.997 \\
\hline RANDOMTREE & 99.19 & 0.992 & 0.989 & 0.995 \\
\hline LWL & 83.88 & 0.937 & 0.825 & 0.860 \\
\hline NAIVEBAYES & 72.66 & 0.903 & 0.665 & 0.911 \\
\hline SVM (SMO) & 88.64 & 0.886 & 0.886 & 0.887 \\
\hline DECISIONTABLE & 96.00 & 0.988 & 0.958 & 0.962 \\
\hline HOEFFDINGTREE & 84.23 & 0.870 & 0.891 & 0.780 \\
\hline BAYESIANNET & 93.96 & 0.984 & 0.955 & 0.923 \\
\hline
\end{tabular}


Figure 1, Figure 2, Figure 3 and Figure 4 show the accuracy, AUC, recall and precision respectively of the top six models evaluated on each of the future test subsets that occur over the subsequent time periods after the ending time of the dataset subset used for model training.

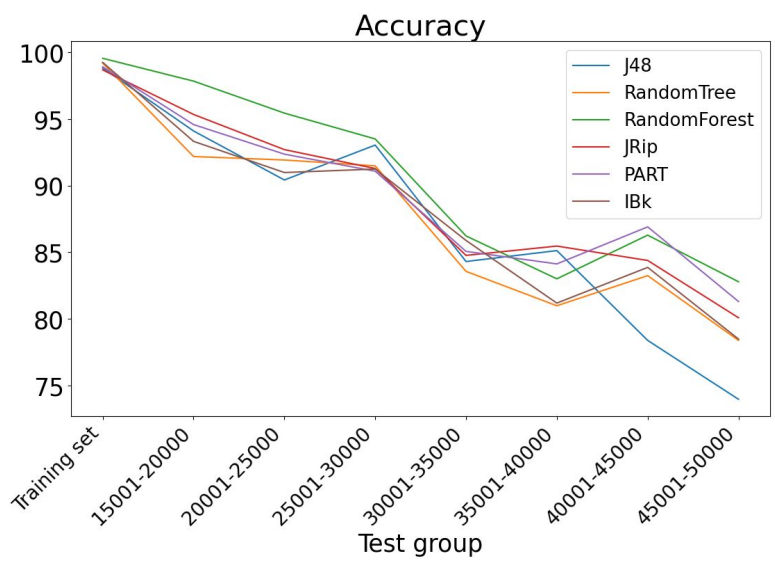

Figure 1. Accuracy of top 6 models trained on initial 15,000 instances using 10 -fold cross validation and then tested on subsequent groups of 5,000 instances

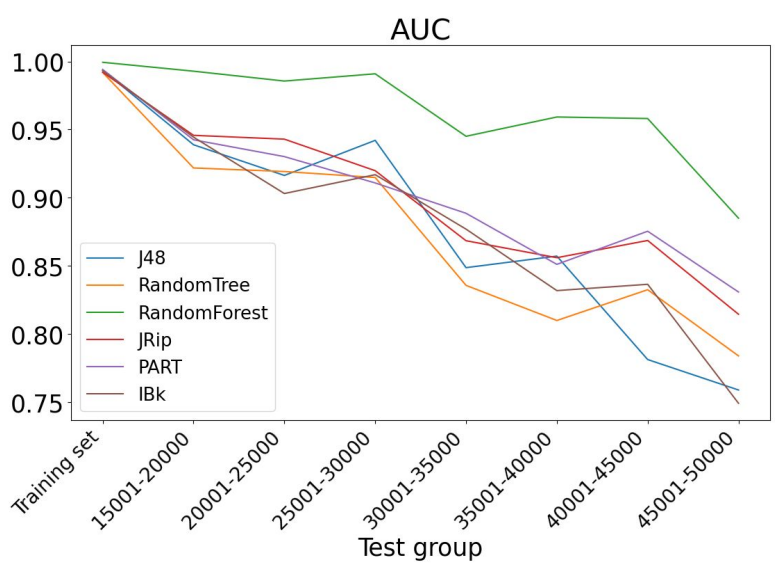

Figure 2. AUC of top 6 models trained on initial 15,000 instances using 10-fold cross validation and then tested on subsequent groups of 5,000 instances

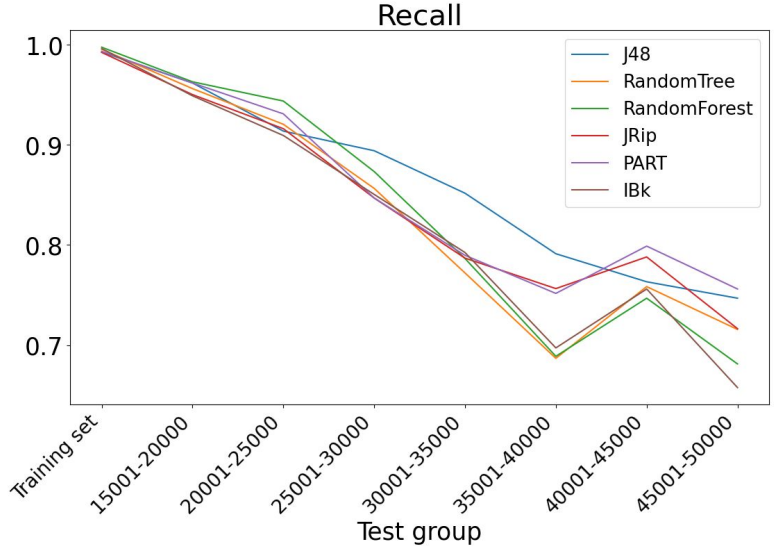

Figure 3. Recall of top 6 models trained on initial 15,000 instances using 10 -fold cross validation and then tested on subsequent groups of 5,000 instances

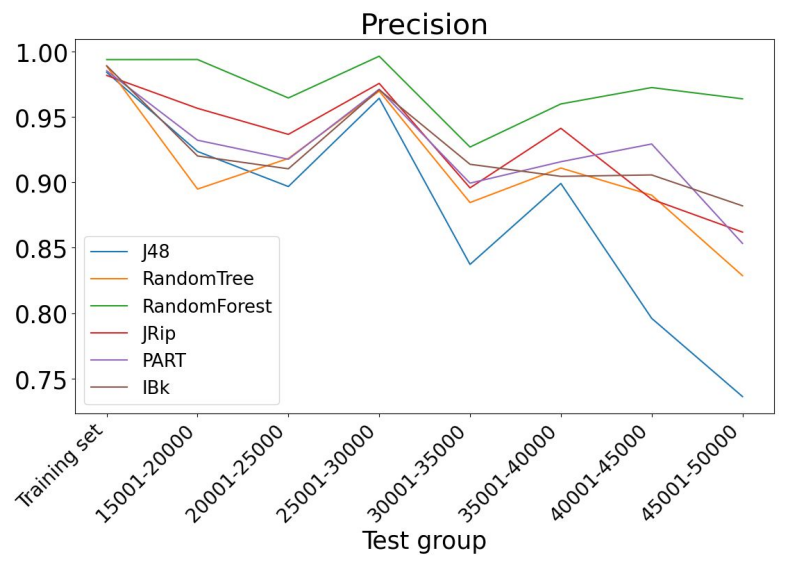

Figure 4. Precision of top 6 models trained on initial 15,000 instances using 10 -fold cross validation and then tested on subsequent groups of 5,000 instances

\subsection{Effect of Length of History of Training Set Used}

In the second group of experiments we considered experiments where the training/model development set includes the most recent executable instances, but we consider the varying durations of preceding history used for training, including ranging from short histories, mid-length histories to all previously seen executables. The group-size was set at 5000 , so the short history was 10,000 ( $2 \mathrm{X} 5000)$, the medium history was 15,000 (3 X 5000) and the long history was all preceding encountered software executables. For 
each of the seven test periods (i.e. instances $15,001-20,000,20,001-25,000 \ldots .45,001-50,000)$ the model has been trained on each of the preceding histories (the prior 10,000, the prior 15,000 and all preceding instances) in each case using 10-fold cross validation. Note that for the first test period (15,0001-20,000), the 3 group (of 5000) history will necessarily be the same as the all preceding instances/groups history.

Figures 5 to 8 show the accuracy, AUC, recall and precision respectively, of the best performing model, Random Forest, trained using 10-fold cross validation on the three historical durations and then evaluated on each test group of 5,000.

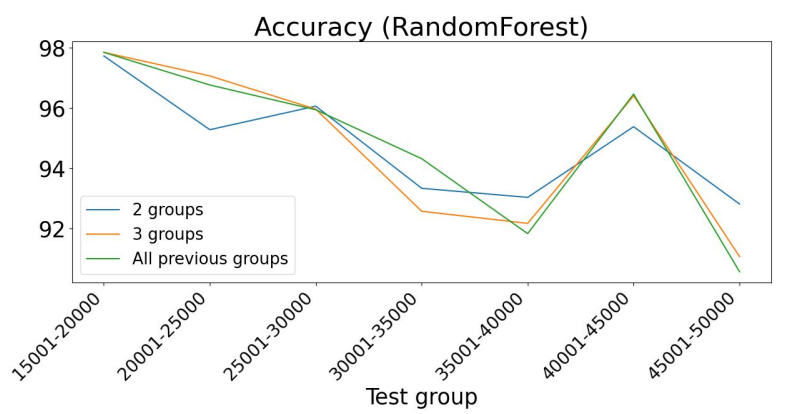

Figure 5. Accuracy of Random Forest trained using 10-fold cross validation on historical training sets of lengths of 2 groups, 3 groups and all previous, and evaluated on the Test Group

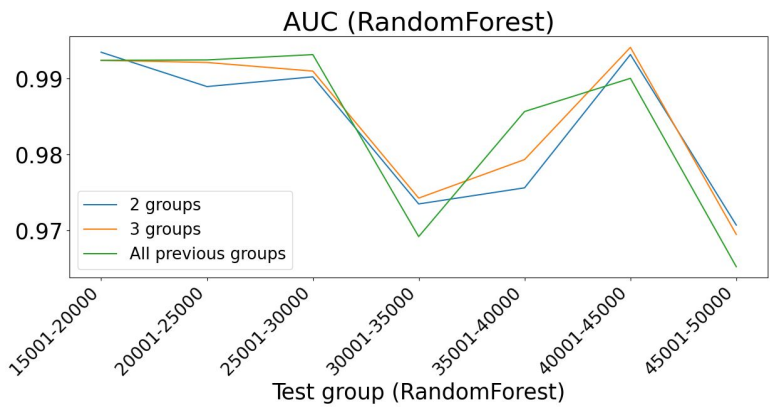

Figure 6. AUC of Random Forest trained using 10-fold cross validation on historical training sets of lengths of 2 groups, 3 groups and all previous, and evaluated on the Test Group

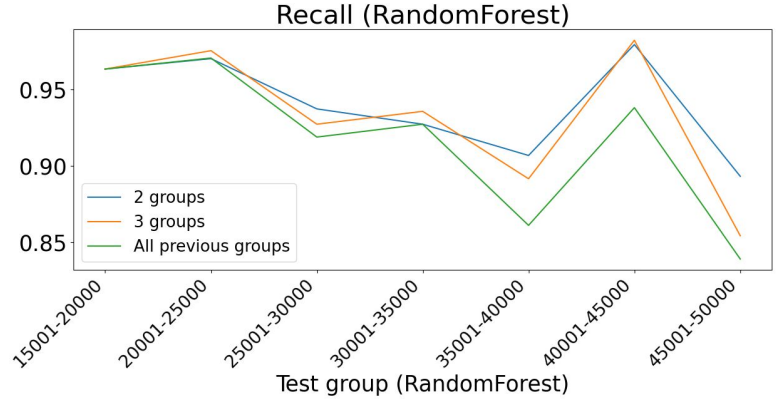

Figure 7. Recall of Random Forest trained using 10-fold cross validation on historical training sets of lengths of 2 groups, 3 groups and all previous, and evaluated on the Test Group

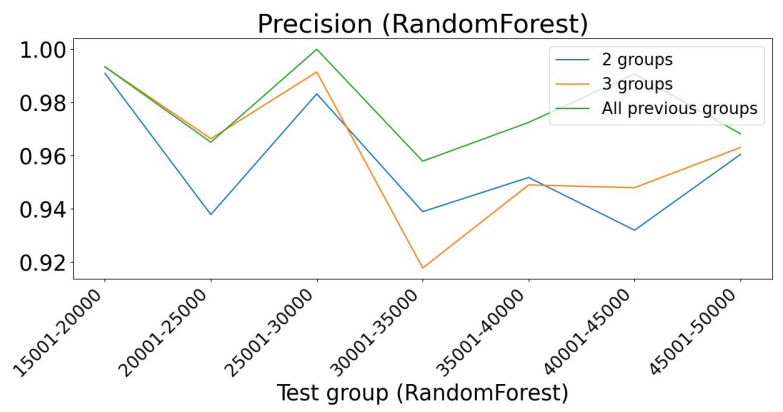

Figure 8. Precision of Random Forest trained using 10-fold cross validation on historical training sets of lengths of 2 groups, 3 groups and all previous, and evaluated on the Test Group

Figures 9 to 12 show the accuracy, AUC, recall and precision respectively, of the Random Tree model, another of the top six models from Experiment 1, in order to consider any comparative or differing aspects.

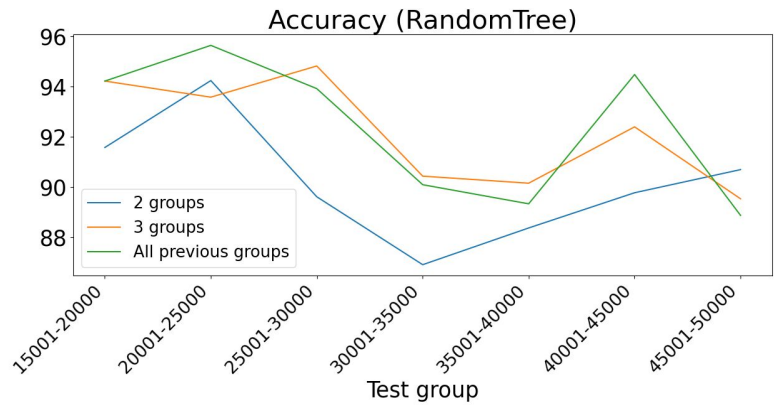

Figure 9. Accuracy of Random Tree trained using 10-fold cross validation on historical training sets of lengths of 2 groups, 3 groups and all previous, and evaluated on the Test Group 


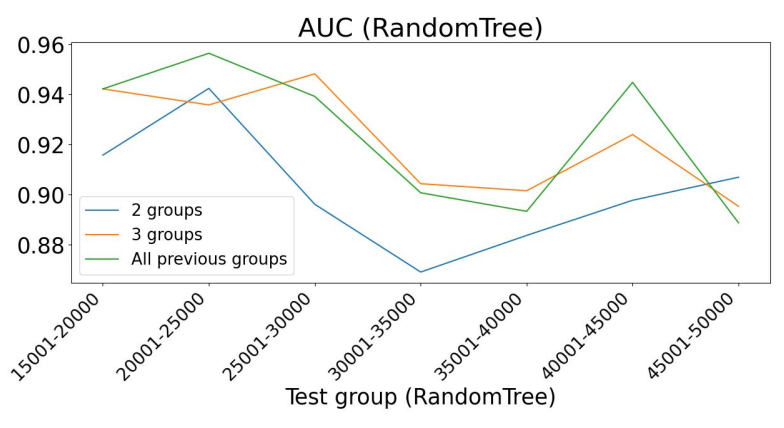

Figure 10. AUC of Random Tree trained using 10-fold cross validation on historical training sets of lengths of 2 groups, 3 groups and all previous, and evaluated on the Test Group

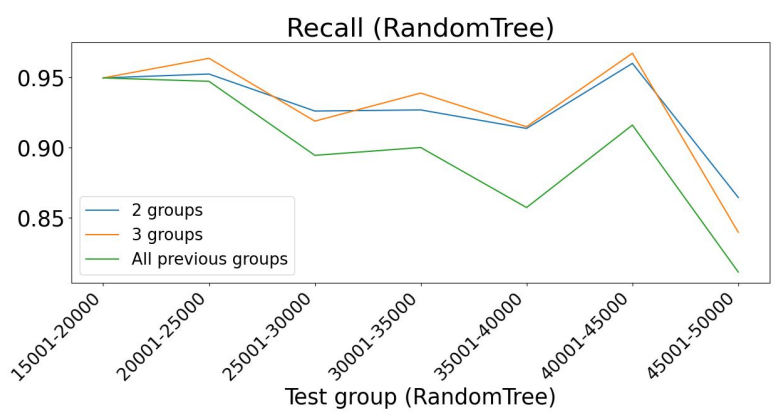

Figure 11. Recall of Random Tree trained using 10-fold cross validation on historical training sets of lengths of 2 groups, 3 groups and all previous, and evaluated on the Test Group

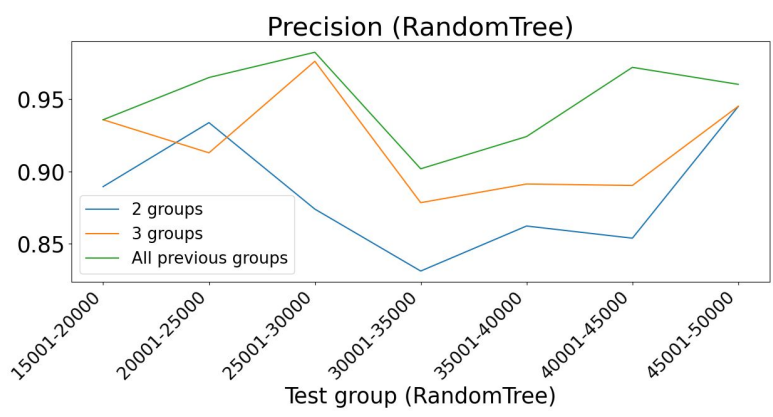

Figure 12. Precision of Random Tree trained using 10-fold cross validation on historical training sets of lengths of 2 groups, 3 groups and all previous, and evaluated on the Test Group

\section{Discussion}

\subsection{Model Performance Deterioration Over Time}

Considering the results of the first set of experiments included in Section 4.1, it is noted that the selected top performing models, all exhibit high accuracy, precision, recall and AUC when trained and evaluated via 10 -fold cross validation on the initial training set (Table 1). See first six rows of Table 1. for the six highest performing models. This mirrors the high discriminative performance found by numerous other studies exploring the performance of machine learning-based malware detection, for example [3-6].

The best performing models achieve an accuracy of $98.6 \%$ or above, and all six of the top performance models achieve an AUC of over 0.99, RandomForest the first in terms of AUC and accuracy, with an AUC of 0.999 .

However, the results from Experiment 1 show a non-monotonic but substantial decrease in model accuracy over time subsequent to training as shown in Figure 1. RandomForest maintains accuracy the best, ending with an accuracy of $82.3 \%$ by the final test set, while C4.5 (J48) has the worst maintenance of accuracy ending with an accuracy of $74.0 \%$.

This highlights an element of ML and AI malware detection models not quantified in a large number of previously published studies, which often conclude with the performance measures obtained by cross-validation evaluation on a whole non-time evolving dataset, without accounting for the reality of practical deployment that the whole dataset, particularly softwares only first encountered in the future, are not available to include in the training set at any given point in time.

Most interestingly, the results also show varied accuracy deterioration rates between the six models studied, but nevertheless notable declines in accuracy with time, with accuracies by the final test sets, representing 2018 data capture, up to $25 \%$ below that exhibited by the models trained via cross-validation on the training set, which represents up to a $25 \%$ decline over the 5 years from training on 2012-2013 data until testing in 2018. RandomForest by contrast, only drops to $82.8 \%$ accuracy over the same 5 years, representing an approximate $16 \%$ decline in accuracy. 
Additionally the somewhat erratic changes in accuracy from one test set to another, with sometimes accuracy increasing for a later test set than for its preceding test set, shows the difficulty for an ML model deployer to know with certainty how well the malware detection will perform, even for a relatively short period into the future. This has implications for model selection, even where models are re-trained at regular intervals.

As shown in Figure 2, it is notable that Random Forest shows a significantly higher maintenance of performance over time than the other high performing models in terms of AUC. By the final test set RandomForest's AUC drops to 0.885 (compared to 0.999 on the development testset), still a high performing AUC, with the next best model being PART with 0.830. KNN is least effective in maintaining AUC performance dropping to 0.750 by the final test set (0.992 initially). To compare with the other identified study of model performance deterioration [11] that has a similar goal to quantify performance deterioration albeit in the different domain of Medicare fraud detection datasets, Random Forest was also found to be the best performing model in terms of AUC for a 2014-15 dataset they used to test a previously trained model. It can also be noted that the systematicness of our current paper is greater than previous studies, considering performance deterioration over a large sequence of future test sets, rather than just one or two future test sets. Maintenance of performance in terms of recall however shows differing results (Figure. 3)

Figure 3 summarizes model recall over time, upon successive test sets, and this overall tends to decline at a relatively constant rate between the six models. By the last test set, some divergence in recall is seen, but with a significant drop for all models, ranging from a recall of approximately 0.756 for PART and dropping to 0.658 for $\mathrm{KNN}$. The counter-trend in recall around the 35001 to 45000 section of the test sets, may be a result of a feature of the dataset collection described in [2], where from January to July 2016 malware collection was suspended, affecting the mix of softwares being inputted to the dataset during that period.

Figure 4 summarizes model precision over time subsequent to the end of the training set period. Performance in terms of precision is relatively erratic over time, and with significant performance differences between the six models. Random Forest again performs the best across the overall range of test sets, and achieves a high precision of 0.964 by the last test set. C4.5 (J48) by comparison achieves the poorest precision by the last test set, with a precision of 0.736 .

It is anticipated, but not yet comprehensively demonstrated or even addressed in this current paper, that the performance deterioration characteristics of ML models trained on software artifacts such as malare/goodware datasets will be more generalizable than models developed for application domains that can be nation or state dependent [12] or even city specific [16].

\subsection{Effect of Duration of Training Set History}

Figures 5 to 8 summarize the effects of the length of the history used in the training set of the ML-based malware detection model considered, in these figures the evaluation is done for Random Forest.

Observations include that in terms of accuracy of the model considered, while the results are mixed and somewhat variable, accuracy is sometimes lower for a shorter history and sometimes higher as per Figure 5. For example for test groups 35,001-40,000 and $45,001-50,000$, the shortest training history (2 groups or 10,000 instances) performs the best.

Additionally, the AUC of the model considered tends to show mixed results in relation to the duration of the training set history as per Figure 6. All previous history/groups shows the lowest performance, rather than the highest as might potentially be expected for the $30,001-35,000,40,001-45,000$ and 45,001-50,000 test groups.

Interestingly the recall of the models considered appears to show a clearer pattern of being higher as the duration of the training set history decreases as per Figure 7. This is an interesting result for malware detection being considered in this study and for this particular PE dataset. A possible explanation for this, is that potentially with a longer history of examples included, there is a greater divergence from the malware examples encountered during the testing period potentially leading to a decreased recall.

Finally the precision of the models considered appears to generally be higher with an increase in duration of the training set used as shown in Figure. 8. As with accuracy and AUC, the shorter duration of the training set, leads to a spike down in precision for some future test set periods. Using the full executable 
history, high precision for Random Forest is maintained, staying about 0.96 at all stages.

Figures 9 to 12, for a slightly lesser performing model from Experiment 1, Random Tree, show similar results in relation to mixed results in relation to accuracy and AUC for length of historical training set being used. It also again surprisingly shows a trend towards higher recall, for a shorter history training set. In terms of precision (Figure. 12), a clearer pattern that a longer training set history, leads to higher precision. In summary, the two different performing models show fairly similar broad characteristics in response to the length of history of the training set.

\subsection{Discussion Summary}

Overall, the results from both groups of experiments highlight the complex relationships between model selection, selection of training set, level and rate of model performance deterioration and evaluation metric used. In terms of model performance maintenance over time subsequent to last training, Random Forest performs the best and shows a significantly higher AUC score for each test set through time, maintaining an AUC of 0.88 on the 2018 test data, executables first seen five years after the training period..

The somewhat unpredictable and hard to explain swings in model performance is suggestive of the uncertainty inherent in machine learning applications in the security domain. They are suggestive of the need for greater assurance safeguards and better understanding of performance deterioration, to limit risks in machine learning-based security applications. Identifying models that demonstrate typically low rates of performance deterioration between any potential re-trainings, and identifying beneficial training period durations for these, have significant practical implications for more robust ML-based malware detection.

\section{Conclusion}

In this paper we have described a systematic analysis of performance maintenance of ML-based malware detection models trained and then tested on a range of data subsets drawn from a large dataset of over 50,000 time-ordered malwares and goodwares, spanning a period from 2012 to 2018. We have considered both model performance deterioration with time elapsed from time of training subset, and also the effect of historical training set duration on the performance of models evaluated on future test sets. Results include interesting observations on the higher and consistent AUC and precision performance of Random Forest over time for the malware detection dataset considered in the study and also the interesting behavior of a number of the models considered, in relation to improved recall as training history duration is decreased rather than increased. The literature review highlights the relative scarcity of studies quantifying $\mathrm{ML}$ and $\mathrm{AI}$ model performance deterioration over time on real-world datasets, not just a scarcity of malware detection performance deterioration studies but across other application domains also.

\section{References}

[1] Malware-goodware dataset: https://github.com/fabriciojoc/brazilian-malware-dataset

[2] F. Ceschin, F. Pinage, M. Castilho, D. Menotti, L.S. Oliveira \& A. Gregio. The need for speed: An analysis of brazilian malware classifers. IEEE Security \& Privacy, 16(6), 2018, 31-41.

[3] Markel, Z., \& Bilzor, M. (2014, October). Building a machine learning classifier for malware detection. In 2014 Second Workshop on Anti-malware Testing Research (WATeR) (pp. 1-4). IEEE.

[4] Kumar, A., Kuppusamy, K. S., \& Aghila, G. (2019). A learning model to detect maliciousness of portable executable using integrated feature set. Journal of King Saud University-Computer and Information Sciences, 31(2), 252-265.

[5] Sami, A., Yadegari, B., Rahimi, H., Peiravian, N., Hashemi, S., \& Hamze, A. (2010, March). Malware detection based on mining API calls. In Proceedings of the 2010 ACM symposium on applied computing (pp. 1020-1025).

[6] Peiravian, N., \& Zhu, X. (2013, November). Machine learning for android malware detection using permission and api calls. In 2013 IEEE 25th international conference on tools with artificial intelligence (pp. 300-305). IEEE.

[7] Sun, Y., Tang, K., Zhu, Z., \& Yao, X. (2018). Concept drift adaptation by exploiting historical knowledge. IEEE transactions on neural networks and learning systems, 29(10), 4822-4832.

[8] Jordaney, R., Sharad, K., Dash, S. K., Wang, Z., Papini, D., Nouretdinov, I., \& Cavallaro, L. (2017). Transcend: Detecting concept drift in malware classification 
models. In 26th \{USENIX\} Security Symposium (\{USENIX\} Security 17) (pp. 625-642).

[9] Galen, C., \& Steele, R. (2020). Performance Maintenance Over Time of Random Forest-based Malware Detection Models. Proceedings of the 11th IEEE Annual Ubiquitous Computing, Electronics \& Mobile Communications Conference, New York, IEEE.

[10] Leevy, J. L., Khoshgoftaar, T. M., Bauder, R. A., \& Seliya, N. (2020). Investigating the relationship between time and predictive model maintenance. Journal of Big Data, 7(1), 1-19.

[11] Leevy, J. L., Khoshgoftaar, T. M., Bauder, R. A., \& Seliya, N. (2019, December). The effect of time on the maintenance of a predictive model. In 2019 18th IEEE International Conference On Machine Learning And Applications (ICMLA) (pp. 1891-1896). IEEE.

[12] Steele, R. J., \& Thompson, B. (2019, January). Data mining for generalizable pre-admission prediction of elective length of stay. In 2019 IEEE 9th Annual Computing and Communication Workshop and Conference (CCWC) (pp. 0127-0133). IEEE.

[13] Hillsgrove, T., \& Steele, R. (2019, March). Utilization of Data Mining for Generalizable, All-admission Prediction of Inpatient Mortality. In 2019 IEEE 2nd International Conference on Information and Computer Technologies (ICICT) (pp. 71-75). IEEE.

[14] M. Hall, E. Frank, G. Holmes, B. Pfahringer, P. Reutemann \& I. Witten. "The WEKA data mining software: an update". ACM SIGKDD explorations newsletter, 11(1), 10-18, 2009.

[15] A. Hanle and B.J. McNeil. "The meaning and use of the area under a receiver operating characteristic (ROC) curve". Radiology, 143(1), 29-36, 1982.

[16] Hillsgrove, T., \& Steele, R. (2019, April). Machine Learning-based Wait-time Prediction for Autonomous Mobility-on-Demand Systems. In Proceedings of 2019 SoutheastCon (pp. 1-7). IEEE. 